\title{
A SUFFICIENT CONDITION FOR EVENTUAL DISCONJUGACY
}

\section{WILLIAM F. TRENCH}

ABSTRACT. It is known that the scalar equation $y^{(n)}+p_{1}(t) y^{(n-1)}+$ $\cdots+p_{n}(t) y=0, t>0, n>1$, is eventually disconjugate if $p_{1}, \ldots, p_{n} \epsilon$ $C[0, \infty)$ and $\int^{\infty} p_{i}(t) \mid t^{i-1} d t<\propto, 1 \leq i \leq n$. This paper presents a weaker integral condition which also implies that the given equation is eventually disconjugate.

A linear differential equation

$$
y^{(n)}+p_{1}(t) y^{(n-1)}+\cdots+p_{n}(t) y=0, \quad t>0 \quad(n>1),
$$

is eventually disconjugate if there is an interval $[a, \infty)$ on which none of its nontrivial solutions has more than $n-1$ zeros, counting multiplicities. From a theorem of Willett [4, Theorem 1.4], (1) is eventually disconjugate if $p_{1}$, $\cdots, p_{n} \in C[0, \infty)$ and

$$
\int^{\infty}\left|p_{i}(t)\right| t^{i-1} d t<\infty, \quad 1 \leq i \leq n
$$

This paper presents a weaker sufficient condition for eventual disconjugacy.

Let $I$ be the set of functions defined for large $t$ and integrable at $\propto$, let $A_{0}$ be the set of functions in $I$ and absolutely integrable at $\infty$, and let $\Phi$ be the set of functions which are positive, nondecreasing and absolutely continuous for large $t$. From Abel's theorem [1, p. 476], $f / \Phi \in I$ if $f \in I$ and $\phi \in \Phi$, and

$$
\int_{t}^{\infty} f(s)(\phi(s))^{-1} d s=o(1 / \phi(s)) .
$$

(In this paper, "O" and "O" refer to behavior as $t \rightarrow \infty_{\text {.) }}$

For $i \geq 1$, define $A_{i}$ as follows: $f \in A_{i}$ if and only if $f \in I$ and there are functions $\phi_{1}, \ldots, \phi_{i}$ in $\Phi$ such that if

$$
f_{0}=f \text {, }
$$

$$
\begin{gathered}
Q_{j}(t)=\int_{t}^{\infty} f_{j-1}(s)\left(\phi_{j}(s)\right)^{-1} d s, \quad 1 \leq j \leq i, \\
f_{j}(t)=t^{-1} \phi_{j}(t) Q_{j}(t), \quad 1 \leq j \leq i,
\end{gathered}
$$

and

$$
g_{j}(t)=\phi_{j}^{\prime}(t) Q_{j}(t), \quad 1 \leq j \leq i
$$

Received by the editors June 7, 1974.

AMS (MOS) subject classifications (1970). Primary 34C10; Secondary 34D05.

Key words and phrases. Eventually disconjugate, linear.

Copyright (c) 1975. American Mathematical Society 
then $f_{0}, \ldots, f_{i-1} \in I$ and $f_{i}, g_{1}, \ldots, g_{i} \in A_{0}$. Finally, define

$$
B_{k-1}=\bigcup_{j=0}^{k-1} A_{j}, \quad k \geq 1 \text {. }
$$

Our main result is

Theorem l. If $p_{1}, \ldots, p_{n} \in C[0, \propto)$ and

$$
t^{k-1} p_{k} \in B_{k-1}, \quad 1 \leq k \leq n
$$

then (1) is eventually disconjugate.

To prove this theorem, we will show that its hypothesis implies that (1) has a fundamental set of solutions $y_{0}, \ldots, y_{n-1}$ such that

$$
y_{r}^{(j)}(t)= \begin{cases}t^{r-j}(1+o(1)) /(r-j) !, & 0 \leq j \leq r, \\ o\left(t^{r-j}\right), & r+1 \leq j \leq n-1 .\end{cases}
$$

If (10) holds, then the Wronskians $W_{r}=W\left(y_{0}, \ldots, y_{r}\right)$ satisfy

$$
W_{r}(t)=1+o(1), \quad 0 \leq r \leq n-1,
$$

and are therefore positive on some interval $[a, \infty)$. Because of this, Pólya's disconjugacy condition [3] implies that (1) is disconjugate on $[a, \infty)$.

(To see that (10) implies (11), observe that a typical term in the expansion of $W_{r}(t)$ according to the definition of determinant is of the form $\pm \prod_{i=0}^{r-1} y_{i}^{\left(j_{i}^{r}\right)}(t)$, where $\left\{j_{0}, \ldots, j_{r-1}\right\}$ is a permutation of $\{0, \ldots, r-1\}$. The product for which $j_{i}=i(0 \leq i \leq r-1)$ equals $1+o(1)$, from (10). Every other product is of the order $\prod_{i=0}^{r-1} O\left(t^{i-j}\right)$, where " $O$ " can be replaced by "o" in at least one factor. Since $\Sigma_{i=0}^{r-1}\left(i-j_{i}\right)=0$, every such product equals o(1).)

We will use the contraction mapping principle to show that $y_{0}, \ldots$. $y_{n-1}$ exist. The subspace $P_{r}\left[t_{0}, \infty\right)$ of $C^{(n-1)}\left[t_{0}, \infty\right)$ consisting of functions such that $y^{(j)}(t)=O\left(t^{r-j}\right), 0 \leq j \leq n-1$, is a Banach space under the norm $\sigma_{r}\left(t_{0} ; y\right)$, where

$$
\sigma_{r}(t: y)=\sum_{j=0}^{n-1} \sup _{s \geq t}\left|s^{j-r} y^{(j)}(s)\right|
$$

With

$$
(M y)(t)=\sum_{k=1}^{n} p_{k}(t) y^{(n-k)}(t)
$$

(cf. (1)), define the mappings $T_{0}, \ldots, T_{n-1}$ by

$$
\left(T_{0} y\right)(t)=1+\int_{t}^{\infty} \frac{(t-s)^{n-1}}{(n-1) !}(M y)(s) d s
$$


and

$$
\begin{aligned}
\left(T_{r} y\right)(t)=\frac{t^{r}}{r !}+\int_{t_{0}}^{t} \frac{(t-\lambda)^{r-1}}{(r-1) !} d \lambda \int_{\lambda}^{\infty} \frac{(\lambda-s)^{n-r-1}}{(n-r-1) !}\left(M_{y}\right)(s) d s, & \\
r & =1, \ldots, n-1 .
\end{aligned}
$$

We will show that (9) implies $T_{r}$ is a contraction mapping of $P_{r}\left[t_{0}, \infty\right)$ into itself if $t_{0}$ is sufficiently large. It will then follow from the contraction mapping principle $[2, \mathrm{p} .11]$ that there is a function $y_{r}$ in $P_{r}\left[t_{0}, \infty\right)$ such that $T_{r} y_{r}=y_{r}$. It is then straightforward to verify that $y_{r}$ satisfies (1) and (10).

Thus, the proof of Theorem 1 is reduced to showing that $T_{r}$ has the stated property. We do this by means of the following lemmas.

Lemma 1. Suppose $f \in A_{i}, h \in C^{(i)}\left[t_{0}, \infty\right)\left(t_{0} \geq 0\right)$, and

$$
h^{(j)}(t)=O\left(t^{-j}\right), \quad 0 \leq j \leq i .
$$

Then the integral

$$
\int_{t}^{\infty} s^{-a} f(s) h(s) d s, \quad a \geq 0, \quad t \geq t_{0},
$$

exists. Moreover, there is a function $m_{i}$, which depends on $f$ but not on $h$ or $t_{0}$, such that

$$
m_{i}(t)=o(1)
$$

and

$$
\begin{array}{r}
\left|t^{a} \int_{t}^{\infty} s^{-a} f(s) h(s) d s\right| \leq m_{i}(t) \sum_{j=0}^{i} \sup _{s \geq t}\left|s^{j} h^{(j)}(s)\right|, \\
\quad 0 \leq a \leq n-1, t \geq t_{0} .
\end{array}
$$

Proof. If $i=0,(16)$ converges absolutely and (17) and (18) hold, with $m_{0}(t)=\int_{t}^{\infty}|f(s)| d s$. If $i \geq 1$, define

$$
h_{0}=h, \quad h_{r}=t h_{r-1}^{\prime}-a h_{r-1}, \quad 1 \leq r \leq i,
$$

and observe that $h_{0}, \ldots, h_{i}$ are bounded because of (15).

Repeated integration by parts yields

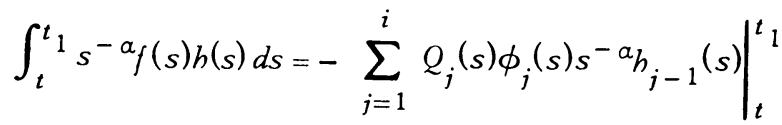

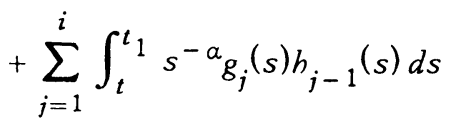

$$
\begin{aligned}
& +\int_{t}^{t} s^{-a} f_{i}(s) h_{i}(s) d s, \quad t_{1} \geq t \geq t_{0},
\end{aligned}
$$


where $\phi_{j}, f_{j}, g_{j}, Q_{j}(1 \leq j \leq i)$ are the functions introduced in defining the class $A_{i}$ (cf. (4)-(7)) and $h_{0}, \ldots, h_{i}$ are as defined in (19). Since $\lim _{t_{1} \rightarrow \infty} Q_{j}\left(t_{1}\right) \phi_{j}\left(t_{1}\right)=0,1 \leq j \leq i$, (by Abel's lemma; cf. (3)), the boundedness of $h_{0}, \ldots, h_{i}$ and the absolute integrability of $f_{i}$ and $g_{1}, \ldots, g_{i}$ at $\propto$ imply that the right side of (20) converges as $t_{1} \rightarrow \infty$. Hence, the integral (16) converges, and it is easy to verify from (20) that (17) and (18) hold, with

$$
m_{i}(t)=K_{i}\left[\int_{t}^{\infty}\left|f_{i}(s)\right| d s+\sum_{j=1}^{i}\left(\left|Q_{j}(t)\right| \phi_{j}(t)+\int_{t}^{\infty}\left|g_{j}(s)\right| d s\right)\right],
$$

where $K_{i}$ is a constant, which does not depend on $h$, such that

$$
\sup _{s \geq t}\left|h_{r}(s)\right| \leq K_{i} \sum_{j=0}^{i} \sup _{s \geq t}\left|s^{j} h^{(j)}(s)\right|, \quad 0 \leq r \leq i, \quad 0 \leq \alpha \leq n-1 .
$$

The existence of $K_{i}$ follows from (15) and (19).

The next lemma follows immediately from (8) and Lemma 1.

Lemma 2. The integral (16) exists if $f \in B_{k-1}, x \in C^{(k-1)}\left[t_{0}, \infty\right)$, and

$$
x^{(j)}(t)=O\left(t^{-j}\right), \quad 0 \leq j \leq k-1 .
$$

Moreover, there is a function $\mu_{k-1}$, which depends on $f$, but not on $h$ or $t_{0}$, such that

$$
\mu_{k-1}(t)=o(1)
$$

and

$$
\begin{array}{r}
\left|t^{a} \int_{t}^{\infty} s^{-a} f(s) h(s) d s\right| \leq \mu_{k-1}(t) \sum_{j=0}^{k-1} \sup _{s \geq t}\left|s^{j} h^{(j)}(s)\right|, \\
\quad 0 \leq \alpha \leq n-1, \quad t \geq t_{0} .
\end{array}
$$

Lemma 3. If the hypotheses of Theorem 1 are satisfied, then the function $z_{\text {, defined by }}$

$$
z_{r}(t)=\int_{t}^{\infty} \frac{(t-s)^{n-r-1}}{(n-r-1) !}\left(M_{y}\right)(s) d s, \quad 0 \leq r \leq n-1,
$$

(cf. (12)) is in $C^{(n-r)}\left[t_{0}, \infty\right)$ if $y \in P_{r}\left[t_{0}, \infty\right)$. Furthermore,

$$
t^{i}\left|z_{r}^{(i)}(t)\right| \leq G_{r}(t) \sigma_{r}(t ; y), \quad 0 \leq i \leq n-r-1, \quad t \geq t_{0},
$$

where

$$
G_{r}(t)=o(1)
$$

and $G_{r}$ depends on the operator $M$, but not on $y$ or $t_{0}$.

Proof. Integrals of the form 


$$
\int_{t}^{\infty} s^{q} p_{k}(s) y^{(n-k)}(s) d s, \quad 0 \leq q \leq n-r-1,
$$

which appear in (23), can be rewritten as

$$
\int_{t}^{\infty} s^{-n+q+r+1} f_{k}(s) x_{k}(s) d s, \text { with } f_{k}(t)=t^{k-1} p_{k}(t)
$$

and

$$
x_{k}(t)=t^{n-k-r} y^{(n-k)}(t) .
$$

Since $f_{k} \in B_{k-1}$ and $x_{k}$ satisfies (21), Lemma 2 implies that (26) exists and, from (22),

$$
\left|t^{n-q-r-1} \int_{t}^{\infty} s^{q} p_{k}(s) y^{(n-k)}(s) d s\right| \leq \mu_{k-1}(t) \sum_{j=0}^{k-1} \sup _{s \geq t}\left|s^{j} x_{k}^{(j)}(s)\right|,
$$

where $\mu_{k-1}$ is as defined in Lemma 2, with $f=f_{k}$. From (27), there is a constant $\lambda_{r k}$ such that

$$
\sum_{j=0}^{k-1} \sup _{s \geq t}\left|s^{j} x_{k}^{(j)}(s)\right| \leq \frac{\lambda_{r k}}{2} \sigma_{r}(t ; y)
$$

for every $y$ in $P_{r}\left[t_{0}, \infty\right)$, and now (24) and (25) follow from (23), (28), (29) and the in equality

$$
\frac{1}{(n-r-i-1) !} \sum_{\nu=0}^{n-r-i-1}\left(\begin{array}{c}
n-r-i-1 \\
\nu
\end{array}\right)=\frac{2^{n-r-i-1}}{(n-r-i-1) !} \leq 2,
$$

if we take

$$
G_{r}(t)=\sum_{k=1}^{n} \lambda_{r k} \mu_{k-1}(t)
$$

Lemm a 3 implies that $T_{r}$, as defined by (13) and (14), maps $P_{r}\left[t_{0}, \infty\right)$ into itself, for any $t_{0} \geq 0$. Moreover, if $y$ and $\tilde{y}$ are both in $P_{r}\left[t_{0}, \infty\right)$, routine estimates based on (13), (14) and (24) yield

$$
\sigma_{r}\left(t_{0} ; T_{r} y-T_{r} \tilde{y} \leq n \tilde{G}_{r}\left(t_{0}\right) \sigma_{r}\left(t_{0} ; y-\tilde{y}\right),\right.
$$

where

$$
\tilde{G}_{r}\left(t_{0}\right)=\sup _{t \geq t_{0}} G_{r}(t)
$$

Because of (25), we can choose $t_{0}$ so that $\tilde{G}_{r}\left(t_{0}\right)<1 / n$, and then (30) implies that $T_{r}$ is a contraction mapping of $P_{r}\left[t_{0}, \propto\right)$ into itself.

The fixed point (function) $y_{r}$ of $T_{r}$ satisfies (1) on $\left(t_{0}, \infty\right)$, and can be extended as a solution of $(1)$ over $(0, \infty)$. To see that $y_{r}$ satisfies $(10)$ for 
$r \leq j \leq n-1$, observe that

$$
y_{r}^{(j)}(t)=\delta_{r j}+\int_{t}^{\infty} \frac{(t-s)^{n-j-1}}{(n-j-1) !}\left(M_{y_{r}}\right)(s) d s, \quad r \leq j \leq n-1,
$$

and apply Lemma 3, with $y=y_{r}$. For $0 \leq j \leq r-1$,

$$
y_{r}^{(j)}(t)=\frac{t^{r-j}}{(r-j) !}+\int_{t_{0}}^{t} \frac{(t-\lambda)^{r-j-1}}{(r-j-1) !} z_{r}(\lambda) d \lambda,
$$

with $z_{r}$ as defined by (23), with $y=y_{r}$. However,

$$
\left|t^{j-r} \int_{t_{0}}^{t}(t-\lambda)^{r-j-1} \gamma_{r}(\lambda) d \lambda\right| \leq t^{-1} \int_{t_{0}}^{t}\left|z_{r}(\lambda)\right| d \lambda
$$

which approaches zero as $t \rightarrow \infty$. (This is obvious if the last integral on the right converges as $t \rightarrow \infty$, and it follows from L'Hospital's rule and Lemma 3 if it diverges.) Thus, $y$, satisfies (10) for $0 \leq j \leq r-1$, and the proof of Theorem 1 is complete.

To the author's knowledge, the classes $A_{1}, A_{2}, \cdots$ have not been previously considered in the literature, and virtually all questions about them are open. For example, is $A_{i-1} \subset A_{i}$ ? How can one construct functions which are in $A_{i}$, but not in $B_{i-1}$ ? Since we cannot answer these questions yet, we will for the present simply exhibit classes of functions which are in $A_{1}$ and $A_{2}$, but not in $A_{0}$. This will show that Theorem 1 implies eventual disconjugacy for some equations which do not satisfy (2).

Theorem 2. Suppose $F$ is continuous and has a bounded antiderivative $F_{1}$ on $[0,, \infty)$, and $\psi$ is absolutely continuous and approaches zero monotonically as $t \rightarrow \infty$. Then the function $f=F \psi$ is in $A_{1}$ if

$$
\int^{\infty} t^{-1}|\psi(t)| d t<\infty
$$

Proof. Take $\phi_{1}=1$ in (5). Then

$$
Q_{1}(t)=\int_{t}^{\infty} F(s) \psi(s) d s=-F_{1}(t) \psi(t)-\int_{t}^{\infty} F_{1}(s) \psi^{\prime}(s) d s,
$$

and our hypotheses imply that $Q_{1}(t)=O(\psi(t))$, and therefore, from $(6), f_{1}(t)=$ $O\left(t^{-1} \psi(t)\right)$, so that (31) implies $f_{1} \in A_{0}$. Since $g_{1}=0$, it follows that $f \in A_{1}$.

As an application of Theorem 2, consider the iterated logarithms:

$$
L_{0}(t)=t, L_{1}(t)=\log t, L_{2}(t)=\log (\log t), \ldots, L_{i}(t)=\log L_{i-1}(t) .
$$

Since $L_{i}^{\prime}(t)=\left[\Pi_{j=0}^{i-1} L_{j}(t)\right]^{-1}, i \geq 1$, the function

$$
\dot{\psi}(t)=\left[L_{i}(t+\rho)\right]^{-\alpha}\left[\prod_{j=1}^{i-1} L_{j}(t+\rho)\right]^{-1}, \quad i \geq 1
$$


(where $\rho$ is a positive constant such that $\psi$ is defined on $[0, \propto)$ ) has the properties required in Theorem 2, provided $\alpha>1$. Theorems 1 and 2 imply, for example, that the equation

$$
y^{(n)}+\frac{t^{-n+1}[\log (\log (t+2 e))]^{-3 / 2} \sin t}{\log (t+2 e)} y=0
$$

is eventually disconjugate if $n \geq 2$. This equation does not satisfy (2).

Theorem 3. Suppose $F$ is continuous on $[0, \infty)$ and has a bounded antiderivative $F_{1}$, which in turn has a bounded antiderivative $F_{2}$. Suppose also that $\psi$ and $\psi^{\prime}$ both tend monotonically to zero as $t \rightarrow \infty$, and $\psi^{\prime}$ is absolute. ly continuous. Then the function $f=F \psi$ is in $A_{2}$.

$$
\text { Proof. From (5) (with } \phi_{1}=1 \text { ), }
$$

$$
Q_{1}(t)=\int_{t}^{\infty} F(s) \psi(s) d s=-F_{1}(t) \psi(t)+F_{2}(t) \psi^{\prime}(t)+\int_{t}^{\infty} F_{2}(s) \psi^{\prime \prime}(s) d s
$$

Our hypotheses imply that the integral on the right equals $O\left(\psi^{\prime}(t)\right)$, so (6) and (33) yield

$$
f_{1}(t)=-F_{1}(t) t^{-1} \psi(t)+A(t) t^{-1} \psi^{\prime}(t)
$$

where $A$ is continuous and bounded on $[0, \infty)$. Letting $\phi_{2}=1$ in (5) yields

$$
\begin{aligned}
Q_{2}(t) & =-\int_{t}^{\infty} F_{1}(s) s^{-1} \psi(s) d s+\int_{t}^{\infty} A(s) s^{-1} \psi^{\prime}(s) d s \\
& =F_{2}(t) t^{-1} \psi(t)+\int_{t}^{\infty} F_{2}(s)\left(s^{-1} \psi(s)\right)^{\prime} d s+\int_{t}^{\infty} A(s) s^{-1} \psi^{\prime}(s) d s
\end{aligned}
$$

Our hypotheses and the boundedness of $A$ imply that each term on the right is $O\left(t^{-1} \psi(t)\right)$; hence (6) and (34) yield

$$
f_{2}(t)=O\left(t^{-2} \psi(t)\right)=o\left(t^{-2}\right)
$$

and therefore $f_{2} \in A_{0}$. Since $g_{1}=g_{2}=0$, it follows that $f \in A_{2}$.

Theorems 1 and 3 imply, for example, that the equation

$$
y^{(n)}+\frac{t^{-n+1} \sin t}{L_{k}(t+\rho)} y=0
$$

is eventually disconjugate if $n \geq 3$ and $L_{k}$ is any iterated logarithm (32) defined on $[\rho, \infty)$. This equation does not satisfy (2).

\section{REFERENCES}

1. T. J. I'a. Bromwich, An introduction to the theory of infinite series, Macmillan, New York and London, 1955. 
2. W. A. Coppel, Stability and asymptotic behavior of differential equations, Heath, Boston, Mass., 1955.

3. G. Pólya, On the mean-value theorem corresponding to a given linear homogeneous differential equation, Trans. Amer. Math. Soc. 24 (1924), 312-324.

4. D. Willett, Disconjugacy tests for singular linear differential equations, SIAM. J. Math. Anal. 2 (1971), 536-545. MR $46 \# 3904$.

DEPARTMENT OF MATHEMATICS, DREXEL UNIVERSITY, PHILADELPHIA, PENNSYLVANIA 19104 